\title{
A REMARK ON THE PRECEDING NOTE BY BOCHNER
}

BY I. J. SCHOENBERG

In the preceding paper in this Bulletin,* S. Bochner has proved the following theorem: If $\phi(t)$ is continuous for $-\infty<t<\infty$ and has the property that the inequality

$$
\left|\sum_{r=1}^{n} c_{r} \phi\left(t_{r}\right)\right| \leqq K \cdot \sup _{-\infty<\xi<\infty}\left|\sum_{r=1}^{n} c_{r} e^{i t_{r} \xi}\right|
$$

holds for any $n$ and for any complex-valued constants $c_{r}$ and real constants $t_{r}$, then

$$
\phi(t)=\int_{-\infty}^{\infty} e^{i t \xi} d \eta(\xi), \text { with } \quad V_{-\infty}^{+\infty}(\eta) \leqq K .
$$

Here is a simple proof of the following modification of the above theorem: If $\phi(t)$ is measurable and the inequality

$$
\left|\int_{-\infty}^{\infty} \phi(t) q(t) d t\right| \leqq K \cdot \max _{\xi}\left|\int_{-\infty}^{\infty} e^{i t \xi} q(t) d t\right|
$$

holds for every $q(t) \subset L$, then there is a function of bounded variation $\eta(\xi)$ such that (2) holds almost everywhere. $\dagger$

For let $A$ be the space of functions $q(t) \subset L$, with

$$
\|q\|=\max _{\xi}\left|\int_{-\infty}^{\infty} e^{i \xi t} q(t) d t\right|,
$$

and let $B$ be the space of functions

$$
\psi(t)=\int_{-\infty}^{\infty} e^{i t \xi} d \eta(\xi)
$$

with $\|\psi\|=V_{-\infty}^{+\infty}(\eta)$. The space $A$ is isometric with the space $A^{\prime}$ of functions

* Vol. 40 (1934), pp. 271-276.

† Compare with the note by F. Riesz, Über Sätze von Stone und Bochner, Acta Szeged, vol. 6 (1933), pp. 184-198, which suggested to me the present remark. 


$$
g(\xi)=\int_{-\infty}^{\infty} e^{i \xi t} q(t) d t,
$$

with $\|g\|=\max _{\xi}|g|$. The space $B$ is isometric with the space $B^{\prime}$ of normalized functions of bounded variation $\eta(\xi)$, with $\|\eta\|=V(\eta)$. For if $q(t) \rightleftarrows g(\xi)$ and also $\psi(t) \rightleftarrows \eta(\xi)$, then $\|q\|=\|g\|$ and $\|\psi\|=\|\eta\|$. Moreover

$$
\int_{-\infty}^{\infty} \psi(t) q(t) d t=\int_{-\infty}^{\infty} g(\xi) d \eta(\xi) .
$$

Since the most general linear operation in $A^{\prime}$ is of the form $\int_{-\infty}^{\infty} g(\xi) d \eta(\xi)$ (note that the functions $g(\xi)$ of our class vanish at infinity), it follows from the isometric relation stated above that the most general linear operation in $A$ is of the form $\int_{-\infty}^{\infty} \psi(t) q(t) d t$, where $\psi(t) \subset B$. Our assumption (3) shows that $\int_{-\infty}^{\infty} \phi(t) q(t) d t$ is a linear operation in $A$; hence there is a function $\psi(t) \subset B$, that is, of the form (2), such that the relation

$$
\int_{-\infty}^{\infty} \phi(t) q(t) d t=\int_{-\infty}^{\infty} \psi(t) q(t) d t
$$

holds for every $q(t) \subset L$; hence $\phi(t)=\psi(t)$ almost everywhere.

Concerning the relationship between the original Theorem (1) and the modified Theorem (3), I might add the following remark. On one hand the modified theorem seems to be more general, since it takes care also of the case when the function $\phi(t)$ is defined or known only almost everywhere, in which case the condition (1) can not be applied. On the other hand, in order to derive the original theorem from the modified one, one has to show directly that from (1), and for $\phi(t)$ continuous, there also follows (3) for any $q(t) \subset L$. It is of course true via both theorems, but there does not seem to be a simple direct proof available.

Institute for Advanced Study 